\title{
PESSOA VIVENDO EM SITUAÇÃO DE RUA SOB UMA ÓTICA DA INVISIBILIDADE: REVISÃO INTEGRATIVA
}

\author{
RESUMO
}

Angelo Aparecido Ninditi ${ }^{1}$

Introdução: O morar na rua no Brasil tem sido associado à drogadição, à vagabundagem, ao fracasso, bem como à periculosidade dos centros urbanos, insegurança, furtos, roubos, à sujeira das ruas, desordem etc. A partir desse imaginário societário, construiu-se uma imagem desqualificante e estigmatizante desses viventes que ressoa negativamente nas práticas cotidianas das pessoas e nas políticas públicas em geral. Revisão Integrativa: A definição de população de rua é difícil. A multiplicidade de condições pessoais, a diversidade de soluções dadas à subsistência e à moradia são fatores que dificultam a formulação de conceitos livres de ambiguidades. Método: O presente artigo trata-se de uma revisão integrativa baseada na pesquisa bibliográfica formulada através de artigos publicados sobre o conhecimento e atuação de profissionais enfermeiros sobre pessoas vivendo em situação de rua. Foram utilizados artigos de plataformas conceituadas como "Bireme, Lilacs, Scielo, Pubmed". Resultados: Foram selecionados um total de 19 artigos científicos, sendo que desse total apenas 17 artigos foram publicados a partir de 2016 e desse total 15 artigos continha classificação na plataforma "Sucupira". Discussão: Pessoas em Situação de Rua (PsR) fazem parte de segmento populacional que vem aumentando consideravelmente nos últimos anos, o qual é caracterizado por condições de extrema pobreza. Considerações Finais: Pessoa em situação de rua fazem parte de segmento populacional que vem aumentando consideravelmente nos últimos anos, o qual é caracterizado por condições extremas pobreza. As pessoas em situação de rua enfrentam, diariamente, situações desafiadoras como violência, privação de direitos e estigma social.

Descritores: enfermagem, situação de rua, vulnerabilidade, invisibilidade social

\section{PERSON LIVING ON THE STREET FROM A VIEW OF INVISIBILITY: INTEGRATIVE REVIEW}

\begin{abstract}
Introduction: Living on the street in Brazil has been associated with drug addiction, vagrancy, failure, as well as the dangerousness of urban centers, insecurity, theft, robberies, dirt on the streets, disorder, etc. From this societal imaginary, a disqualifying and stigmatizing image of these living beings was built, which resonates negatively in people's daily practices and in public policies in general. Integrative Review: Defining homeless people is difficult. The multiplicity of personal conditions, the diversity of solutions given to subsistence and housing are factors that make it difficult to formulate concepts free of ambiguities. Method: This article is an integrative review based on bibliographic research formulated through published articles on the knowledge and performance of professional nurses on people living on the streets. Articles from renowned platforms such as "Bireme, Lilacs, Scielo, Pubmed" were used. Results: A total of 19 scientific articles were selected, of which only 17 articles were published from 2016 and of this total 15 articles were classified on the "Sucupira" platform. Discussion: Homeless People (PsR) are part of a population segment that has increased considerably in recent years, which is characterized by conditions of extreme poverty. Final Considerations: Homeless people are part of a population segment that has increased considerably in recent years, which is characterized by extreme poverty. People living on the streets face daily challenging situations such as violence, deprivation of rights and social stigma.
\end{abstract}

Descriptors: nursing, homelessness, vulnerability, social invisibility

${ }^{1}$ Graduado em enfermagem pela Universidade Salgado Oliveira - Universo BH, 2016.

E-mail: angeloninditi07@gmail.com 


\section{Introdução}

O morar na rua no Brasil tem sido associado à drogadição, à vagabundagem, ao fracasso, bem como à periculosidade dos centros urbanos, insegurança, furtos, roubos, à sujeira das ruas, desordem etc. A partir desse imaginário societário, construiu-se uma imagem desqualificante e estigmatizante desses viventes que ressoa negativamente nas práticas cotidianas das pessoas e nas políticas públicas em geral. A ausência de um conhecimento sistematizado - quantitativo e qualitativo - sobre a vida de quem vive nas ruas, agregado à cultura do assistencialismo, à invisibilidade, à intolerância, ao preconceito contra o diferente e ao desinteresse estatal, manteve o escopo das ações governamentais apenas no âmbito reativo aos problemas imediatos: albergue, comida e roupa (RIOS, A. G; SEIXAS, C. T; et al, 2021).

Morar na rua e ser mulher amplia estigmas que são socialmente compartilhados: é a transversalidade de gênero reforçando outras exclusões. Significa enfrentar formulações socialmente produzidas a partir de uma visão geral que se tem das pessoas que vivem nas ruas, e do que seria "o melhor" para elas (RIOS, A. G; SEIXAS, C. T; et al, 2021).

Entende-se que travestis e mulheres transexuais que vivem em situação de rua são pessoas em conjuntura de vulnerabilidade e duplamente excluídas socialmente. As histórias de vida narradas têm em comum, e quase na mesma sequência, conflitos familiares relacionados com as questões de gênero, seguidos pela saída de casa, prostituição e situação de rua (MENDES, L. G; JORGE. A. O. \& PILECCO, F. B; 2019).

O direito fundamental à saúde foi instituído no Brasil em 1988 por meio da promulgação da Constituição Federal (CF/88). Muito além da ausência de doença, a saúde é compreendida como um estado multideterminado, que guarda íntima relação com as condições de vida e acesso aos bens públicos e sociais de indivíduos e grupos populacionais de determinada sociedade (VALLE, F. A. A. L; FARAH, B. F. \& CARNEIRO JUNIOR, N; 2020)

As pessoas que fazem dos espaços das ruas como moradia expõem vulnerabilidades complexas, apresentando heterogêneas necessidades e demandas para a manutenção de suas vidas. Nesse contexto, necessitam de serviços sociais e de saúde mais adequados às suas peculiaridades (VALLE, F. A. A. L; FARAH, B. F. \& CARNEIRO JUNIOR, N; 2020). Historicamente, as políticas sociais visam responder às demandas advindas das relações contraditórias determinadas pelas lutas de classes, raça, gênero e etnia (MENDES, L. G; JORGE. A. O. \& PILECCO, F. B; 2019). Os conceitos relacionados com o gênero, uma 
vez estabelecidos como um conjunto de referências e normas, estruturam tanto a percepção quanto a organização concreta e simbólica das relações sociais. Dessa forma, podese dizer que as convenções hegemônicas que associam o sexo biológico à identidade de gênero regulam e produzem mecanismos de coerção e submissão a padrões normatizados socialmente o que, além de gerar diferentes posições de poder na vida em sociedade, conduzem ou podem conduzir a situações de vulnerabilidade social (MENDES, L. G; JORGE. A. O. \& PILECCO, F. B; 2019).

Sob o ponto de vista da saúde, é alta a prevalência de doenças como Tuberculose, HIV/AIDS, dermatites, comorbidades psiquiátricas e uso abusivo de drogas. $\mathrm{O}$ alcoolismo e outras drogas geralmente estão relacionados tanto com a manutenção das pessoas na rua, quanto com a exposição delas à violência (BRITO, C. \& SILVA, L. N; 2022).

Nos espaços urbanos, a pessoa em situação de rua é frequentemente impedida de entrar em locais como transporte coletivo, serviços de saúde, outros órgãos públicos. Também é recorrente a tendência de responsabilizá-la por seus próprios problemas, sem levar em conta os contextos que os produziram (BRITO, C. \& SILVA, L. N; 2022).

A tuberculose (TB) é principal causa de morte entre as doenças infecciosas em todo o mundo. Sabe-se que a pobreza é uma das causas da TB, e que o adoecimento gera ainda mais pobreza, mantendo ativo um ciclo que envolve indivíduos, famílias e comunidades (MENDES, L. G; JORGE. A. O. \& PILECCO, F. B; 2019). Alguns fatores são conhecidos pela literatura como potenciais influenciadores no desfecho do tratamento da tuberculose, sendo eles: sexo, idade, escolaridade, forma da $\mathrm{TB}$, agravos associados principalmente AIDS, diabetes e doenças mentais, uso de álcool, tabaco ou drogas, dentre outros. Porém há uma carência de informações sobre como as populações privadas de liberdade ou em situação de rua no Brasil podem se relacionar com o desfecho da doença (MENDES, L. G; JORGE. A. O. \& PILECCO, F. B; 2019)..

\section{Revisão Integrativa}

\subsection{Cenário e definição de população de rua}

A definição de população de rua é difícil. A multiplicidade de condições pessoais, a diversidade de soluções dadas à subsistência e à moradia são fatores que dificultam a formulação de conceitos livres de ambiguidades. (SILVA ROSA, A. CAVICCHIOLI, M. G. S. \& BRETTAS, A. C. P, 205). É constituída por pessoas que não têm moradia, pernoitam em 
albergues, nos logradouros da cidade, em casas abandonadas, cemitérios, carcaças de veículos, terrenos baldios ou em depósitos de papelão e sucata.

Morar na rua configura-se como um problema global evidenciado tanto em nações desenvolvidas como nas que estão em desenvolvimento, principalmente em centros urbanos de médio e de grande porte. Entretanto, constitui-se um desafio quantificar esta população e estabelecer definições sobre o que consiste estar em situação de rua, considerando-se as diferentes realidades de cada país. Na União Europeia, o percentual desta população tem apresentado um aumento na última década, sendo que apenas Finlândia e Holanda apresentaram uma redução nesse período (BISCOTTO, P. R; et al, 2016).

Pessoas em Situação de Rua fazem parte de segmento populacional que vem aumentando consideravelmente nos últimos anos, o qual é caracterizado por condições de extrema pobreza. Estima-se que, em março de 2020, havia 221.869 Pessoas em Situação de Rua no Brasil, com maior concentração em municípios de grande porte. As Pessoas em Situação de Rua enfrentam, diariamente, situações desafiadoras como violência, privação de direitos e estigma social. Essa realidade tem impacto direto na saúde e na qualidade de vida dessas pessoas, pois possui maior probabilidade de resultar em condições crônicas, distúrbios mentais e transtornos por uso de substâncias psicoativas (XIMENESI, M. A. M; et al, 2021).

Pessoas em situação de rua têm difícil acesso aos benefícios garantidos pelas políticas públicas, inclusive os recursos de saúde. Para facilitar o acesso ao sistema de saúde, o Consultório na Rua (CR), fundamentado nas diretrizes definidas na Política Nacional da Atenção Básica (PNAB), foi concebido para ser a porta de entrada aos serviços de saúde, propiciando atenção integral e longitudinal, coordenando o cuidado e prestando atenção à saúde da população em situação de rua in loco ou em outros pontos de atenção da rede de saúde (KAMI, M. T. M; LAROCCA; et al, 2016).

A vida na rua expõe quem vive nela, a riscos que exigem das equipes de saúde uma abordagem específica para essa população (KAMI, M. T. M; et al, 2016).

A vida nas ruas faz com que as pessoas, cotidianamente, se deparem com uma diversidade de situações que envolvem desafios, como o acesso à alimentação e ao transporte, dificuldades financeiras, vícios e estigma social. Embora em menor número, a mulher em situação de rua se torna mais vulnerável por viver em um contexto permeado por preconceitos, violência, desigualdade de gênero e de direitos sociais (BISCOTTO, P. R; et al, 2016).

As pessoas em situação de rua são um grupo heterogêneo que tem em comum a pobreza extrema, vínculos familiares interrompidos ou fragilizados e a inexistência de 
moradia convencional regular (SILVA, A. B; et al, 2020). Por outro lado, essas mesmas pessoas possuem suas particularidades, modos de vida e formas de poder (relações) que as fazem resistir em contextos de repressão, estigmas e políticas públicas frágeis. Logo, a rua também é um espaço de morada e de trabalho informal para essas pessoas: guardadores de carro, descarregadores de carga, catadores de papel ou latinhas, entre outros. Além disso, é um território de afeto e proteção para a superação das dificuldades de viver com poucos recursos e dos estigmas que sofrem (SILVA, A. B; et al, 2020).

O cuidado é intrínseco à condição humana. Embora o termo seja frequentemente utilizado na discussão sobre a integralidade e a humanização das práticas de saúde, sua definição ainda é imprecisa pela complexidade que lhe é inerente. De modo geral, o cuidado pode ser entendido como a interação entre duas ou mais pessoas, com o objetivo de aliviar o sofrimento e alcançar o bem-estar, mediado por conhecimentos voltados para essa finalidade, e, muitas vezes, processa-se por condutas normativas reduzidas a procedimentos, prescrições, normatizações, em detrimento de um cuidado que valorize os projetos de vida do outro (SILVA, I. C. N, et al, 2018). O contexto da rua é onde inúmeras pessoas buscam ser acolhidas, amparadas e abrigadas, embora sejam constantemente submetidas a condições insalubres e a aglomerados humanos, bem como à privação de alimento e água, à exposição a variações climáticas e a situações de violência (SILVA, I. C. N, et al, 2018).

Com o advento da Constituição, em 1988, a saúde passou a ser um direito de cidadania no Brasil, assegurado através do SUS, cujo objetivo é oferecer uma cobertura universal, integral e equitativa por meio de redes organizadas de serviços, sob gestão compartilhada entre a esfera federal, estadual e municipal do governo. No entanto, o baixo financiamento público decorrente da agenda neoliberal da década de 1990 fomentou uma expansão importante de planos privados de saúde. Tanto do ponto de vista do financiamento como da prestação dos serviços, o sistema de saúde brasileiro pode ser considerado híbrido, constituído por três subsistemas: o SUS financiado com recursos do Estado e de acesso universal com ênfase na atenção primária; um subsistema privado, podendo ter fins lucrativos ou não, mantido com recursos públicos e privados; e o subsistema suplementar composto de diversos tipos de planos privados, que também recebe subsídios fiscais (BORYSOW, I. C, 2017).

\subsection{Mulheres em situação de rua}


No Brasil, a discussão sobre a situação de vida e saúde das mulheres e de seus bebês, no contexto da rua, ocupou o debate social, político e jurídico, especialmente a partir de 2012, fortemente associado ao uso de drogas. O tema alcançou a mídia, sugerindo uma relação direta entre o uso de drogas e a situação de rua, (re)produzida pelo discurso moral da “dependência química", em que a droga seria a causa da passagem à vida na rua (SANTOS, G. C; BAPTISTA, T. W. F. \& CONSTANTINO, P; 2021). A situação dessas mulheres e de seus bebês denuncia um mal-estar social ancorado nas históricas e desiguais relações de gênero, classe e raça que incidem sobre corpos específicos: mulheres, em sua grande maioria pretas e pobres, cuja história de vida é marcada por violências estruturais e institucionais. Trata-se de mulheres que vivem em um profundo contexto de desamparo e desproteção social, desafiando normas instituídas sobre o que é ser mulher e mãe, provocando respostas do Estado (SANTOS, G. C; BAPTISTA, T. W. F. \& CONSTANTINO, P; 2021)

Historicamente, as políticas sociais visam responder às demandas advindas das relações contraditórias determinadas pelas lutas de classes, raça, gênero e etnia. (MENDES, $L$. G; JORGE. A. O. \& PILECCO, F. B; 2019). Apesar de não superarem a desigualdade, podese dizer que as políticas públicas alteram o padrão de distanciamento entre as classes sociais na medida em que instituem sistemas de proteção social.

\subsection{A saúde da população de rua}

A tuberculose ainda é, no século XXI, um importante problema de saúde pública no mundo, apesar de ser uma doença tratável e curável. Em 2015, houve 10,4 milhões de novos casos e 1,4 milhão de óbitos pela doença, posicionando-a entre as principais causas de morte no mundo. (SANTOS, A. C. E; et al, 2021) No Brasil, o enfrentamento da tuberculose está previsto em diversos planos de ação governamentais e nas iniciativas nacionais para atingir os Objetivos de Desenvolvimento Sustentável das Nações Unidas. Apesar de ter apresentado melhora nos indicadores, o Brasil ainda ocupava o $20^{\circ}$ lugar entre os países com maior carga da doença e o $19^{\circ}$ lugar quanto à coinfecção HIV/ tuberculose no mundo em 2015, o que o fez permanecer na lista de nações de ações prioritárias da Organização Mundial de Saúde (OMS) para o quadriênio 2016-2020.

O controle da tuberculose no Brasil encontra obstáculos diante da dificuldade histórica em difundir informações sobre a doença de forma mais eficiente, das crises econômicas com impactos negativos nos índices de pobreza, da má distribuição de riquezas, 
do precário processo de urbanização, do aumento da infecção por HIV, de insatisfatórios níveis de nutrição, de condições sanitárias precárias e do aumento das populações em vulnerabilidade. (SANTOS, A. C. E; et al, 2021) Nesse último grupo destacam-se as pessoas em situação de rua, um grupo de risco especial para a tuberculose por sofrer extrema exclusão social, acesso precário aos serviços de saúde e diversas situações de violência e discriminação, além de possuir vínculos familiares fragilizados ou inexistentes.

A tuberculose (TB) é um dos problemas graves de saúde pública para todo o mundo, principalmente para os 22 países mais afetados, que contribuem com cerca de $80 \%$ dos casos. O Brasil ocupa a $16^{\text {a }}$ posição em número absoluto de casos. (ALECRIM, T. F. A; et al, 2016).

A TB é uma doença socialmente determinada; portanto, o modo de viver na rua reforça a vulnerabilidade à doença por diversos motivos decorrentes, tais como: falta de moradia, condições precárias de sono, repouso e alimentação, uso abusivo de álcool e outras drogas, luta pela sobrevivência, dificuldade para realizar o autocuidado, entre outros (HINO, P; et al, 2021)

O grupo mais vulnerável à TB tem sido o da população em situação da rua, em decorrência da pobreza, falta de emprego, do desconhecimento da doença, da coinfecção por HIV, ausência de residência fixa, baixa imunidade, imigração e do uso de substâncias psicoativas. Nesse contexto, essa população tem 48 a 67 vezes mais chances de adoecer por TB quando comparada à população em geral, ficando evidente que esse grupo é o mais acometido pela doença. Outrossim, apresenta maior necessidade de cuidado em saúde, fazendo-se necessários serviços mais articulados em suas práticas para garantir a atenção integral e equânime a esses indivíduos. Assim, trabalhar com pessoas que vivem nessa situação exige estabelecimento de vínculos que possibilitem humanizar o encontro, para tanto é necessário compreender sem julgar, respeitar e estabelecer limites (ALECRIM, T. F. A; et al, 2016).

\subsection{Enfermagem no cuidado da pessoa em situação de rua}

A equipe de enfermagem, composta por enfermeiras/os, técnicas/os e auxiliares de enfermagem, é detentora de grande quantitativo de profissionais de saúde no Sistema Único de Saúde (SUS), estando presente na composição da maioria dos serviços, nos três níveis de atenção. (ZANDOMINGO, M. N. P; et al, 2020) O seu trabalho está relacionado ao processo 
de cuidado, colocando-a em evidência nos serviços de saúde, em contato direto e frequente com as PSR. A atuação da equipe de enfermagem junto às PSR na RAPS varia de acordo com as características do serviço e suas atribuições no contexto da equipe multidisciplinar.

População em Situação de Rua (PSR) e entendida como um grupo populacional heterogêneo com características relacionadas à pobreza extrema, vínculos familiares interrompidos ou fragilizados, sem moradia convencional regular, utilizando logradouros públicos e/ou áreas degradadas, de forma temporária ou permanente, como moradia e sustento, podendo ou não fazer uso de unidades de acolhimento para pernoite ou moradia provisória (KOOPMANS, F. F; et al, 2019).

As precárias condições de vida nas ruas deixam as PSR mais propensas a sofrerem lesões e problemas de saúde relacionados ao estilo de vida, que inclui nutrição fraca por conta da alimentação incerta, exposição aos elementos do clima e situações de violências, como assaltos e espancamentos. $\mathrm{O}$ acesso à água e a sua qualidade, a privação de sono e de afeição também são elementos que afetam a saúde dessas pessoas. Ademais, apresentam significativas demandas relacionadas a transtornos mentais e ao uso de álcool ou outras drogas, que potencializam suas vulnerabilidades e requerem cuidados em saúde (ZANDOMINGO, M. N. P; et al, 2020).

Higiene precária, mau cheiro e efeito de drogas lícitas e ilícitas pode se constituir barreira para o acolhimento de PSR em serviços de saúde. (ZANDOMINGO, M. N. P; et al, 2020)É comum também rigidez no agendamento de atendimentos, restrição nos horários disponíveis, além da exigência de documentos de identidade, comprovante de endereço e Cartão Nacional de Saúde (Cartão SUS) como condição para atendimento e realização de procedimentos.

As especificidades da vida em situação de rua, associadas à complexidade de fatores, tornam as pessoas vulneráveis a vários agravos sociais e de saúde que desafiam profissionais, como enfermeiras/os, técnicas/os de enfermagem, médicas/ os, assistentes sociais, odontólogas/os, técnicas/os em saúde bucal, psicólogas/os, agentes comunitários de saúde, terapeutas ocupacionais e agentes sociais, dos diversos setores e serviços da sociedade (SILVA, I. C. N; et al, 2018).

Estudos trazem que os serviços e profissionais de saúde tem pouca experiência e conhecimento em "acolher" pessoas em situação de rua e atender as suas necessidades, gerando precariedade do acolhimento. (KOOPMANS, F. F; et al, 2019) Podendo ocorrer devido a características estigmatizantes desta populacao, como odor e uso de drogas e álcool, 
dificuldade de acesso para agendamento de consultas e inflexibilização de horários, dificuldades na Rede de Apoio para internação, aumentando assim sua invisibilidade

No Brasil, foram criados mecanismos políticos para implementação de políticas e ações de saúde para a pessoa em situação de rua, como a Política Nacional para a População em Situação de Rua (2009) e o Manual sobre Cuidado a Saúde junto a População em Situação de Rua (2012) ambos buscando a ampliação do acesso e a qualidade de atenção à saúde, com políticas publicas especificas e mecanismos de cuidado, como os Consultórios na Rua (CnaR) (KOOPMANS, F. F; et al, 2019).

O Sistema Único de Saúde (SUS) se configura como sistema público responsável por oferecer ações e serviços de saúde de forma universal, integral e equânime à população brasileira. (CARDOSO, A. C; et al, 2018) No que se refere à atenção à população em situação de rua, o Ministério da Saúde do Brasil lançou, em 2011, a Política Nacional de Atenção Básica, instituindo o Consultório na Rua (CnaR) como um de seus dispositivos, com o objetivo de atender aos problemas e necessidades desse grupo social e ofertar, de maneira mais oportuna, ações e serviços de saúde.

Cabe, portanto, às equipes de Consultório na Rua $(\mathrm{eCR})$ prestar atenção à saúde em um cenário diferenciado e dinâmico, que desafia cotidianamente os profissionais de saúde que assistem os usuários em situações que fogem do programado. Para driblar as dificuldades, adversidades e surpresas encontradas nessa realidade complexa da rua, o trabalho em saúde é organizado em uma perspectiva multiprofissional e interdisciplinar. Nesse contexto, espera-se que a enfermagem, como parte da equipe multiprofissional, possa manter relações que possibilitem o compartilhamento do cuidado visando uma atenção integral à pessoa em situação de extrema vulnerabilidade social (CARDOSO, A. C; et al, 2018).

O profissional de saúde entende processo de trabalho como um conjunto de saberes, instrumentos e meios, tendo como sujeitos os profissionais que se organizam para produzir serviços de modo a prestar assistência individual e coletiva para obtenção de produtos e resultados decorrentes de sua prática (CARDOSO, A. C; et al, 2018).

A vida na rua expõe quem vive nela, a riscos que exigem das equipes de saúde uma abordagem específica para essa população. Por se tratar de uma proposta inovadora de atenção a pessoas em situação de rua, o trabalho dos profissionais que integram as equipes do $\mathrm{CR}-\mathrm{eCR}$, apresenta facetas ainda não completamente reveladas por outros autores. (KAMI, M. T. M; et al, 2016) O processo de trabalho em saúde pode ser desdobrado em finalidade, meios e instrumentos e objeto, no qual dois tipos de saberes estão presentes: saberes instrumentais e saberes ideológicos 


\section{Método}

O presente artigo trata-se de uma revisão integrativa baseada na pesquisa bibliográfica formulada através de artigos publicados sobre o conhecimento e atuação de profissionais enfermeiros sobre pessoas vivendo em situação de rua. Foram utilizados artigos de plataformas conceituadas como "Bireme, Lilacs, Scielo, Pubmed".

Em virtude da quantidade crescente e da complexidade de informações na área da saúde, tornou-se imprescindível o desenvolvimento de artifícios, no contexto da pesquisa cientificamente embasada, capazes de delimitar etapas metodológicas mais concisas e de propiciar, aos profissionais, melhor utilização das evidências elucidadas em inúmeros estudos. Nesse cenário, a revisão integrativa emerge como uma metodologia que proporciona a síntese do conhecimento e a incorporação da aplicabilidade de resultados de estudos significativos na prática (SOUZA,M. T; SILVA, M. D. \& CARVALHO, R; 2010).

O método em xeque constitui basicamente um instrumento da Prática Baseada em Evidências (PBE). (SOUZA,M. T; SILVA, M. D. \& CARVALHO, R; 2010) A PBE, cuja origem atrelou-se ao trabalho do epidemiologista Archie Cochrane, caracteriza-se por uma abordagem voltada ao cuidado clínico e ao ensino fundamentado no conhecimento e na qualidade da evidência. Envolve, pois, a definição do problema clínico, a identificação das informações necessárias, a condução da busca de estudos na literatura e sua avaliação crítica, a identificação da aplicabilidade dos dados oriundos das publicações e a determinação de sua utilização para o paciente. (SOUZA,M. T; SILVA, M. D. \& CARVALHO, R; 2010 A revisão integrativa, nesse âmbito, em virtude de sua abordagem metodológica, permite a inclusão de métodos diversos, que têm o potencial de desempenhar um importante papel na PBE em enfermagem. Dessa forma, é fundamental diferenciá-la das linhas de estudos existentes.

A presente pesquisa baseou-se em artigos publicados no período de 2016 a 2022. Dentre os artigos revisados há publicações anteriores a 2016 que foram utilizadas no corpo do texto para construir a revisão integrativa levando em consideração ao pioneirismo e relevância dos autores sobre o assunto mencionado ao longo do trabalho. Foi utilizado nessa pesquisa os seguintes descritores: enfermagem, situação de rua, vulnerabilidade e invisibilidade social. Foram encontrados 1492 artigos com os descritores supracitados em plataformas confiáveis já mencionadas. Quando mencionados individualmente enfermagem foi descrito 1400 descrições, vulnerabilidade 03, situação de rua 01, invisibilidade social 07. Associando os 
descritores ao termo "enfermagem": vulnerabilidade tiveram 23 artigos descritos, situação de rua tiveram 41 artigos mencionados e situação de rua tiveram 41 artigos. Dos 1492 artigos encontrados para a produção desta pesquisa integrativa, apenas 19 artigos foram utilizados no corpo do texto por terem sido publicadas em um período de 05 anos e com classificação Qualis entre A e B. Ressaltando que foram utilizados dois artigos inferiores a estes anos por sua relevância e pioneirismo, sendo uma publicação de 2005 e a outra de 2010.

\section{Resultados}

Foram selecionados um total de 19 artigos científicos, sendo que desse total apenas 17 artigos foram publicados a partir de 2016 e desse total 15 artigos continha classificação na plataforma "Sucupira". Os 04 artigos não classificados, foram publicados a partir do ano de 2020. O Qualis afere a qualidade de artigos entre outros tipos de produção, a partir da análise da qualidade dos veículos de divulgação, ou seja, periódicos científicos. A classificação de periódicos é realizada pelas áreas de conhecimento/atuação e passa por processo anual de atualização. Esses veículos são enquadrados em estratos indicativos da qualidade: A1; A2; B1; B2; B3; B4; B5 e C. Sendo A1 indicando a melhor qualidade de um artigo a $\mathrm{C}$ que indica um artigo sem qualidade ou equivalente a nota zero.

\begin{tabular}{|c|c|c|c|}
\hline \multicolumn{4}{|c|}{ Quadro 01: Representação dos Artigos Avaliados pelo Sistema Qualis } \\
\hline \multirow[t]{2}{*}{ Ordem } & \multirow[t]{2}{*}{ Título do Periódico } & $\begin{array}{c}\text { Campo de } \\
\text { Avaliação } \\
\text { dos Artigos }\end{array}$ & $\begin{array}{l}\text { Conceito } \\
\text { Qualis }\end{array}$ \\
\hline & & $2016-2022$ & $2013-2016$ \\
\hline 01 & $\begin{array}{l}\text { Experiência dos profissionais de saúde no cuidado da } \\
\text { pessoa com tuberculose em situação de rua. }\end{array}$ & Enfermagem & A2 \\
\hline 02 & $\begin{array}{l}\text { Compreensão da vivência de mulheres em situação de } \\
\text { rua. }\end{array}$ & Enfermagem & A2 \\
\hline 03 & $\begin{array}{l}\text { Atenção à saúde de pessoas em situação de rua: estudo } \\
\text { comparado de unidades móveis em Portugal, Estados } \\
\text { Unidos e Brasil. }\end{array}$ & Enfermagem & B1 \\
\hline 04 & $\begin{array}{l}\text { População em situação de rua: estigmas, preconceitos } \\
\text { e estratégias de cuidado em saúde. }\end{array}$ & Enfermagem & B1 \\
\hline 05 & $\begin{array}{llll}\text { Desafios e potencialidades do trabalho } & \text { de } \\
\text { Enfermagem em Consultório na Rua. }\end{array}$ & Enfermagem & A1 \\
\hline 06 & Tuberculose na população de rua: revisão sistemática. & Enfermagem & A1 \\
\hline 07 & $\begin{array}{l}\text { Saberes ideológicos e instrumentais no processo de } \\
\text { trabalho no Consultório na Rua. }\end{array}$ & Enfermagem & A1 \\
\hline & $\mathrm{O}$ viver na rua: revisão integrativa sobre cuidados a & Enfermagem & $\mathrm{A} 2$ \\
\hline
\end{tabular}




\begin{tabular}{|c|c|c|c|}
\hline 08 & moradores de rua. & & \\
\hline 09 & $\begin{array}{l}\text { Proteção social e produção do cuidado a travestis e a } \\
\text { mulheres trans em situação de rua no município de } \\
\text { Belo Horizonte (MG). }\end{array}$ & Enfermagem & $\mathrm{B} 2$ \\
\hline 10 & $\begin{array}{l}\text { A produção do comum como estratégia de cuidado } \\
\text { para usuários complexos: uma cartografia com } \\
\text { mulheres em situação de rua. }\end{array}$ & Enfermagem & B1 \\
\hline 11 & $\begin{array}{l}\text { Análise e comparação dos desfechos do tratamento de } \\
\text { tuberculose na população em situação de rua e na } \\
\text { população geral do Brasil. }\end{array}$ & Enfermagem & Não avaliado \\
\hline 12 & $\begin{array}{l}\text { O processo saúde-doença-cuidado e a população em } \\
\text { situação de rua. }\end{array}$ & Enfermagem & Não avaliado \\
\hline 13 & $\begin{array}{l}\text { O processo saúde-doença-cuidado e a população em } \\
\text { situação de rua. }\end{array}$ & Enfermagem & Não avaliado \\
\hline 14 & $\begin{array}{l}\text { Desvelando a cultura, o estigma e a droga enquanto } \\
\text { estilo de vida na vivência de pessoas em situação de } \\
\text { rua. }\end{array}$ & Enfermagem & B1 \\
\hline 15 & $\begin{array}{l}\text { Representações sociais do cuidado em saúde de } \\
\text { pessoas em situação de rua. }\end{array}$ & Enfermagem & $\mathrm{A} 2$ \\
\hline 16 & Revisão integrativa: o que é e como fazer. & Enfermagem & $\mathrm{B} 2$ \\
\hline 17 & $\begin{array}{l}\text { As vivências na rua que interferem na saúde: } \\
\text { perspectiva da população em situação de rua. }\end{array}$ & Enfermagem & $\mathrm{B} 2$ \\
\hline 18 & $\begin{array}{l}\text { Atividades de vida e diagnósticos de enfermagem na } \\
\text { população de rua. }\end{array}$ & Enfermagem & Não avaliado \\
\hline 19 & $\begin{array}{l}\text { Pobreza, fome e abandono: representações da equipe } \\
\text { de enfermagem sobre pessoas em situação de rua. }\end{array}$ & Enfermagem & A2 \\
\hline
\end{tabular}

Fonte: Ninditi, Angelo Aparecido - 2022.

O quadro 01 representa os 19 artigos selecionados de acordo com o método de inclusão escolhido, sendo de acordo com os descritores . Os artigos selecionados foram publicados entre 2016 e 2022, sendo que as principal área de atuação foi: Enfermagem. Dos 19 artigos selecionados para realização deste artigo foram encontradas 15 avaliações com notas de A1 a B3. O quantitativo de artigos publicados por enfermeiro é muito menor do que se esperava para um tema tão importante e de relevância social. A qualidade dos artigos produzidos por enfermeiro é bastante discutível no que tange a qualidade e a qualidade de produções. Não havendo possibilidade de confirmar se as poucas produções realizadas e de baixa qualidade seria um déficit acumulado desde a academia ou acúmulo de funções e carga horária excessiva de enfermagem, tornando a maioria produções/artigos inviáveis para fontes de pesquisas. Pode-se levar em consideração o estigma e o descaso do poder público a respeito desta população que é invisível aos olhares da sociedade. Fato é que a maioria dos artigos ainda que em pequena quantidade, traz um escopo de preconceito e tabus a serem quebrados.

Percebe-se que ainda estamos longe de uma sociedade acolhedora, com visão holística para perceber o outro para além das tralhas que carregam, de uma autoimagem afeiçoada, que percebe o outro para com uma estrutura humana. Devendo perceber esses 
indivíduos como pessoa, como gente, como produtores de sentimento e de força de trabalho. È necessário ter um olhar de profundidade humana e de respeito mesmo que a mazela impere.

Segue o quadro 02 demonstrando o quantitativo de avaliações na respectiva área de atuação.

\section{Quadro 02: Quantitativo de Avaliação Qualis}

\begin{tabular}{|c|c|c|c|}
\hline Campo de atuação & & & \\
\hline Enfermagem & Níveis & A1 & 01 \\
\hline & & $\mathrm{A} 2$ & 07 \\
\hline & & B1 & 04 \\
\hline & & B2 & 03 \\
\hline & & B3 & 00 \\
\hline & & B4 & 00 \\
\hline & & B5 & 00 \\
\hline & & $\mathrm{C}$ & 00 \\
\hline & & $\begin{array}{c}\text { Não } \\
\text { avaliado }\end{array}$ & 04 \\
\hline
\end{tabular}

Fonte: Ninditi, Angelo Aparecido - 2022

Esse quadro representativo explicita a baixa produtividade de artigos científicos na única área de atuação que se preocupou a ater-se como fonte de produção e de especulação. Pode-se perceber eu apenas 01 artigo teve nota máxima, 07 artigos com a segunda nota melhor; 04 artigos bons, 03 artigos regulares e 04 artigos se quer pode ser mencionados em termos de avaliação. Particularmente acredito que tenham sido publicados a partir de 2020 e que não houve tempo para uma devida avaliação. Todavia pela leitura os acrescento como sendo artigos de grande valia para o meio acadêmico na construção de uma sociedade civil mais justa.

Gráfico 01: Tabela representativa da avaliação Qualis dos artigos analisados

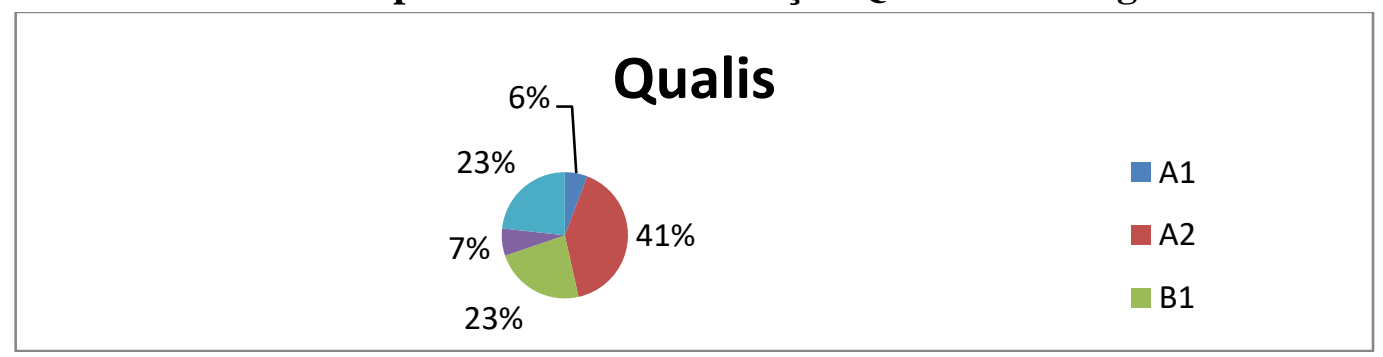

Fonte: Ninditi, Angelo Aparecido - 2022 


\section{Discussão}

Pessoas em Situação de Rua (PsR) fazem parte de segmento populacional que vem aumentando consideravelmente nos últimos anos, o qual é caracterizado por condições de extrema pobreza. Estima-se que, em março de 2020, havia 221.869 PsR no Brasil, com maior concentração em municípios de grande porte (XIMENESI, M. A. M; et al, 2021).

Neste cenário, a assistência a PsR apresenta-se como desafio aos profissionais e sistemas de saúde para garantir acessibilidade aos serviços e equidade das ações. Diante disso, conhecer o contexto dessa população, identificar suas particularidades e deficiências é uma das estratégias para ampliar a promoção da saúde desse grupo vulnerável. Com isso, a enfermagem se destaca por participar da prestação de cuidados diretos a estes indivíduos nos diversos níveis de atenção à saúde, dispondo de ações organizadas e processos de trabalho com base na sistematização da assistência. Ao identificar as necessidades de saúde desta população a partir do processo de enfermagem, é possível estabelecer as ligações necessárias para a implementação de ações efetivas para promoção da saúde (XIMENESI, M. A. M; et al, 2021).

Com o advento da Constituição, em 1988, a saúde passou a ser um direito de cidadania no Brasil, assegurado através do SUS, cujo objetivo é oferecer uma cobertura universal, integral e equitativa por meio de redes organizadas de serviços, sob gestão compartilhada entre a esfera federal, estadual e municipal do governo. No entanto, o baixo financiamento público decorrente da agenda neoliberal da década de 1990 fomentou uma expansão importante de planos privados de saúde. Tanto do ponto de vista do financiamento como da prestação dos serviços, o sistema de saúde brasileiro pode ser considerado híbrido, constituído por três subsistemas: o SUS financiado com recursos do Estado e de acesso universal com ênfase na atenção primária; um subsistema privado, podendo ter fins lucrativos ou não, mantido com recursos públicos e privados; e o subsistema suplementar composto de diversos tipos de planos privados, que também recebe subsídios fiscais (BORYSOW, I. C; CONILL, E. M. \& FURTADO, J. P; 2017).

As estratégias em saúde para as pessoas em situação de rua surgiram de experiências municipais entre os anos 1980 e 2000, algumas relacionadas à atenção primária e outras voltadas aos usuários de substâncias psicoativas em situação de rua. (BORYSOW, I. C; CONILL, E. M. \& FURTADO, J. P; 2017). A partir de 2007, o Ministério de Desenvolvimento Social articulou-se com outros ministérios (Ministério das Cidades, da 
Educação, Saúde, Justiça, Trabalho e Emprego, Secretaria Especial de Direitos Humanos e Defensoria Pública da União), com trabalhadores da área e movimentos populares, o que resultou na produção da Política Nacional para Inclusão Social da População em Situação de Rua

A vida nas ruas faz com que as pessoas, cotidianamente, se deparem com uma diversidade de situações que envolvem desafios, como o acesso à alimentação e ao transporte, dificuldades financeiras, vícios e estigma social. BISCOTTO, P. R; JESUS, M. C. P; et al, 2016) Embora em menor número, a mulher em situação de rua se torna mais vulnerável por viver em um contexto permeado por preconceitos, violência, desigualdade de gênero e de direitos sociais

Com o aumento do número de pessoas em situação de rua no Brasil e ao mesmo tempo iniciativas como a instituição da Política Nacional para a População em Situação de Rua (PNPSR), os gestores públicos dos municípios de maior porte começaram a delinear estratégias de identificação e abordagem com as demandas desse grupo social. (CARDOSO, A. C; et al, 2018) O Sistema Único de Saúde (SUS) se configura como sistema público responsável por oferecer ações e serviços de saúde de forma universal, integral e equânime à população brasileira. No que se refere à atenção à população em situação de rua, o Ministério da Saúde do Brasil lançou, em 2011, a Política Nacional de Atenção Básica, instituindo o Consultório na Rua (CnaR) como um de seus dispositivos, com o objetivo de atender aos problemas e necessidades desse grupo social e ofertar, de maneira mais oportuna, ações e serviços de saúde

Na busca por apreender a complexidade do fenômeno das drogas e suas relações com o cuidado, seu uso e as recentes Políticas Públicas referentes ao tema têm trazido para a cena de discussão a população de rua, chamando a atenção também para os territórios de uso de drogas dessa população, como as cracolândias. Esses locais da cidade são a face dos invisíveis, que desmascaram o ainda distanciamento das políticas públicas sociais e de inclusão voltados a essa população, que acaba ficando à mercê de ações pontuais de limpeza do espaço urbano. (SILVA, A. B; et al, 2020) As pessoas em situação de rua são um grupo heterogêneo que tem em comum a pobreza extrema, vínculos familiares interrompidos ou fragilizados e a inexistência de moradia convencional regular.

O grupo mais vulnerável à tuberculose tem sido o da população em situação da rua, em decorrência da pobreza, falta de emprego, do desconhecimento da doença, da coinfecção por HIV, ausência de residência fixa, baixa imunidade, imigração e do uso de substâncias psicoativas (ALECRIM, T. F. A; et al, 2016). Nesse contexto, essa população tem 
48 a 67 vezes mais chances de adoecer por TB quando comparada à população em geral, ficando evidente que esse grupo é o mais acometido pela doença. Outrossim, apresenta maior necessidade de cuidado em saúde, fazendo-se necessários serviços mais articulados em suas práticas para garantir a atenção integral e equânime a esses indivíduos. Assim, trabalhar com pessoas que vivem nessa situação exige estabelecimento de vínculos que possibilitem humanizar o encontro, para tanto é necessário compreender sem julgar, respeitar e estabelecer limites (ALECRIM, T. F. A; et al, 2016).

Apesar das diferentes causas possíveis para a rua ser adotada como lar, seus moradores partilham inúmeras características. São todos muito pobres, pessoas para as quais algumas das instituições básicas da sociedade - propriedade privada, família, mercado deixaram de propiciar as estratégias usuais de sobrevivência. A trajetória de vida que os levou às ruas desenha, na maioria das vezes, uma sequencia de fatos e fracassos pessoais e desamparo institucional (SILVA ROSA, A; CAVICCHIOLI, M. G. S. \& BRÊTAS, A. C. P; 2005).

Se a exclusão e o desamparo os igualam frente aos olhares da sociedade de uma forma geral, alguns fatores os diferenciam: os motivos que os levaram para a rua, o tempo de permanência nela e o grau de vínculos familiares existentes. A interface desses contribui para classificar o "povo de rua" em três situações distintas: ficar na rua (circunstancialmente), estar na rua (recentemente) e ser da rua (permanentemente). "Ficar na rua" caracteriza transitoriedade, a pessoa possui ainda um projeto de vida e mantém fortes vínculos familiares; "estar na rua" implica na diminuição do contato com a família e o estabelecimento de novos vínculos na rua; "ser da rua" traz em si a identidade e identificação com a própria rua, que passa a ser o lugar de referência e espaço de relações - o corpo se modifica, bem como as

formas de conviver e ver o mundo (SILVA ROSA, A; CAVICCHIOLI, M. G. S. \& BRÊTAS, A. C. $P ; 2005)$.

\section{Considerações Finais}

Pessoa em situação de rua fazem parte de segmento populacional que vem aumentando consideravelmente nos últimos anos, o qual é caracterizado por condições extremas pobreza. As pessoas em situação de rua enfrentam, diariamente, situações desafiadoras como violência, privação de direitos e estigma social. Essa realidade tem impacto direto na saúde e na qualidade de vida dessas pessoas, pois possui maior 
probabilidade de resultar em condições crônicas, distúrbios mentais e transtornos por uso de substâncias psicoativas.

A assistência a pessoas em situações de rua apresenta-se como desafio aos profissionais e sistemas de saúde para garantir acessibilidade aos serviços e equidade das ações. Conhecer o contexto dessa população, identificar suas particularidades e deficiências é uma das estratégias para ampliar a promoção desse grupo vulnerável. Com isso, a enfermagem se destaca por participar da prestação de cuidados diretos a estes indivíduos nos diversos níveis de atenção à saúde, dispondo de ações organizadas de trabalho com base na sistematização da assistência.

Independentemente da realidade social e de saúde das regiões no Brasil, a população em situação de rua apresenta piores desfechos relacionados à tuberculose em comparação com a população geral. A rua precisa ser entendida como um território de cuidado construído sob uma ótica estrutural singular, onde vivem sujeitos com autonomia. Para tanto, é necessário romper a ótica biomédica e estimular a criação de vínculos de confiança e respeito entre os sujeitos e as equipes de saúde. Sabe-se que as equipes conhecidas como Consultórios na Rua são a frente de trabalho para o atendimento dessa população.

Apesar do esforço dos profissionais de saúde, a fragilidade na articulação entre os setores envolvidos na assistência dessa população torna-se, em alguns casos, um empecilho para a efetividade do atendimento e para o cumprimento das metas pactuadas mundialmente. Da mesma forma, logicamente, são necessários crescimento econômico, redução das desigualdades socioeconômicas e políticas sociais eficientes e equânimes para que todas as pessoas tenham suas necessidades supridas, inclusive as de moradia.

O cuidado é intrínseco à condição humana. Embora o termo seja frequentemente utilizado na discussão sobre a integralidade e a humanização das práticas de saúde, sua definição ainda é imprecisa pela complexidade que lhe é inerente. De modo geral, o cuidado pode ser entendido como a interação entre duas ou mais pessoas, com o objetivo de aliviar o sofrimento e alcançar o bem-estar, mediado por conhecimentos voltados para essa finalidade, e, muitas vezes, processa-se por condutas normativas reduzidas a procedimentos, prescrições, normatizações, em detrimento de um cuidado que valorize os projetos de vida do outro.

O número de pessoas em situação de rua está em ascensão no Brasil e em vários outros países, revelando os extremos de desigualdade e exclusão social no mundo. O contexto da rua é onde inúmeras pessoas buscam ser acolhidas, amparadas e abrigadas, embora sejam constantemente submetida a condições insalubres e a aglomerados humanos, bem com à 
privação de alimento e água, à exposição a variações climáticas e a situações de violência. No contexto de rua muitos acabam envolvendo-se com álcool e outras drogas, estão vulneráveis a doenças crônicas, psiquiátricas e infectocontagiosas, como afecções de pele, infestações por piolhos, tuberculose e infecções sexualmente transmissíveis.

\section{Referências Bibliográficas}

ALECRIM, T. F. A; MITANO, F. A; REIS, A; ROOS, C. M; PALHA, P. F. \& PROTTI-ZANATTA, S. T. Experiência dos profissionais de saúde no cuidado da pessoa com tuberculose em situação de rua. Revista Escola de Enfermagem USP. 2016; 50 (5): 808-815. DOI: http://dx.doi.org/10.1590/S0080$\underline{623420160000600014}$

BISCOTTO, P. R; JESUS, M. C. P; SILVA, M. H; OLIVEIRA, D. M. \& MERIGHI, M. A. B. Compreensão da vivência de mulheres em situação de rua. Revista Escola de Enfermagem USP. 2016; 50 (5): 750-756. DOI: http://dx.doi.org/10.1590/S0080-623420160000600006

BORYSOW, I. C; CONILL, E. M. \& FURTADO, J. P. Atenção à saúde de pessoas em situação de rua: estudo comparado de unidades móveis em Portugal, Estados Unidos e Brasil. Ciência \& Saúde Coletiva, 22(3): 879890, 2017. DOI: http://dx.doi.org/10.1590/1413-81232017223.25822016

BRITO, C. \& SILVA, L. N. População em situação de rua: estigmas, preconceitos e estratégias de cuidado em saúde. Ciência \& Saúde Coletiva, 27(1): 151-160, 2022, DOI: http://dx.doi.org/10.1590/1413$\underline{81232022271.19662021}$

CARDOSO, A. C; SANTOS, D. S; MISHIMA, S. M; DOS ANJOS, D. S. C; JORGE, J. S. \& SANTANA, H. P. Desafios e potencialidades do trabalho de Enfermagem em Consultório na Rua. Revista Latino-Americana Enfermagem. 2018;26:e3045. DOI: https://doi.org/10.1590/1518-8345.2323.3045

HINO, P; YAMAMOTO, T. T; BASTOS, S. H; BERALDO, A. A; FIGUEIREDO, T. M. R. M. \& BERTOLOZZI, M. R. Tuberculose na população de rua: revisão sistemática. Revista Escola Enfermagem: USP. 2021; 55: e03688. DOI: https://doi.org/10.1590/S1980-220X2019039603688

KAMI, M. T. M; LAROCCA, L. M; M. M. N. C; PIOSIADLO, L. C. M. \& ALBUQUERQUE, G. S. Saberes ideológicos e instrumentais no processo de trabalho no Consultório na Rua. Revista Escola de Enfermagem USP · 2016; 50 (3): 442-449. DOI: http://dx.doi.org/10.1590/S0080-623420160000400010

KOOPMANS, F. F; DAHER, D. V; ACIOLI, S; SABOIA, V. M; RIBEIRO, C. R. B. \& SILVA, C. S. S. L. O viver na rua: revisão integrativa sobre cuidados a moradores de rua. Revista Brasileira Enfermagem 72 (1), Jan-Feb 2019. DOI: https://doi.org/10.1590/0034-7167-2017-0653

MENDES, L. G; JORGE. A. O. \& PILECCO, F. B. Proteção social e produção do cuidado a travestis e a mulheres trans em situação de rua no município de Belo Horizonte (MG). Saúde Debate: Rio De Janeiro, v. 43, n. ESPECIAL 8, p. 107-119, 2019. DOI: http://dx.doi.org/10.1590/0103-11042019S808

RIOS, A. G; SEIXAS, C. T; CRUZ, K. T; SLOMP JUNIOR, H; MERHY, E. E. \& SANTIAGO, S. M. A produção do comum como estratégia de cuidado para usuários complexos: uma cartografia com mulheres em situação de rua. Ciência \& Saúde Coletiva, 26 (8): 3077-3086, 2021. DOI: http://dx.doi.org/10.1590/1413$\underline{81232021268.12972020}$

SANTOS, A. C. E; BRUNFENTRINKER, C; PENA, L. S; SARAIVA, S. S. \& BOING, A. F. Análise e comparação dos desfechos do tratamento de tuberculose na população em situação de rua e na população geral do Brasil. Jornal Brasileiro de Pneumologia. 2021; 47(2): e20200178 DOI: https://dx.doi.org/10.36416/1806$\underline{3756 / \mathrm{e} 20200178}$ 
SANTOS, G .C; BAPTISTA, T. W. F. \& CONSTANTINO, P. "De quem é esse bebê?": desafios para o direito à maternidade de mulheres em situação de rua. Caderno de Saúde Pública. 2021; 37 (5): e00269320. DOI: http://dx.doi.org/10.1590/0102-311X00269320

SILVA ROSA, A; CAVICCHIOLI, M. G. S. \& BRÊTAS, A. C. P. O processo saúde-doença-cuidado e a população em situação de rua. Revista Latino-am Enfermagem. 2005 julho-agosto; 13(4):576-82. DOI: http://dx.doi.org/10.159/s0104-11692005000400017

SILVA, A. B; OLSCHOWSKY, A; WETZEL, Christine; SILVA, T. J. \& PAVANI, F. M. Desvelando a cultura, o estigma e a droga enquanto estilo de vida na vivência de pessoas em situação de rua. Ciência \& Saúde Coletiva, 25 (10): 3713-3721, 2020. DOI: http://dx.doi.org/10.1590/1413-812320202510.36212018

SILVA, I. C. N; SANTOS, M. V. S; CAMPOS, L. M. C; SILVA D. O; PORCINO, C. A. \& OLIVEIRA, J. F. Representações sociais do cuidado em saúde de pessoas em situação de rua. Revista Escola de Enfermagem USP. 2018; 52: e03314. DOI: http://dx.doi.org/10.1590/S1980-220X2017023703314

SOUZA,M. T; SILVA, M. D. \& CARVALHO, R. Revisão integrativa: o que é e como fazer. Einstein (São Paulo) 8 (1). Jan-Mar 2010. DOI: https://doi.org/10.1590/S1679-45082010RW1134

VALLE, F. A. A. L; FARAH, B. F. \& CARNEIRO JUNIOR, N. As vivências na rua que interferem na saúde: perspectiva da população em situação de rua. Saúde Debate: Rio de Janeiro, v. 44, n. 124, p. 182-192, 2020. DOI: http://dx.doi.org/10.1590/0103-1104202012413

XIMENESI, M. A. M; GOMES, J. S; CAVALCANTE, F. M. L; GALINDO NETO, N. M; CAETANO, A; BARROS \& L. M. Atividades de vida e diagnósticos de enfermagem na população de rua. Revista de enfermagem UERJ. Rio de Janeiro, 2021; 29: e56956. DOI: http://dx.doi.org/10.12957/reuerj.2021.56956

ZANDOMINGO, M. N. P; OLIVEIRA, J. F; SILVA, D.O; PORCINO ,C; SUTO, C. S. S. \& OLIVEIRA, D. S. Pobreza, fome e abandono: representações da equipe de enfermagem sobre pessoas em situação de rua. Revista Brasileira Enfermagem. 2020;73(Suppl 1): 1 DOI: http://dx.doi.org/10.1590/0034-7167-2019-0338 e20190338 